\title{
A Novel Error Compensation Method of Five-axis Flank Milling of Ruled Surface by Modifying Tool Path
}

Gaiyun He ( $\nabla$ hegaiyun@tju.edu.cn )

Tianjin University

Chenglin Yao

Tianjin University

Yicun Sang

Tianjin University

Yichen Yan

Tianjin University

\section{Research Article}

Keywords: Error compensation, Five-axis flank milling, Ruled surface, Tool path modification, Multi-peaks Gaussian fitting

Posted Date: July 16th, 2021

DOI: https://doi.org/10.21203/rs.3.rs-713428/v1

License: (c) (i) This work is licensed under a Creative Commons Attribution 4.0 International License. Read Full License 


\title{
A novel error compensation method for five-axis flank milling of ruled surface by modifying tool path
}

\author{
Gaiyun He*, Chenglin Yao, Yicun Sang, Yichen Yan \\ (Key Laboratory of Mechanism Theory and Equipment Design of Ministry of Education, Tianjin \\ University, Tianjin, 300072, China) \\ *Corresponding author. E-mail address: hegaiyun@tju.edu.cn
}

\begin{abstract}
Five-axis flank milling is widely used in the aerospace and automotive industry. However, diverse sources of errors prevent the improvement of machining accuracy. This paper proposes a novel error compensation method for five-axis flank milling of ruled surface by modifying the original tool path according to the error distribution model. The method contains three steps: First, the errors at the middle of the straight generatrix on the machined surface are calculated according to error distribution, and the corresponding normal vectors are obtained by geometric calculation. Second, multipeaks Gaussian fitting method is utilized to make connections between parameters in the original tool path and error distribution. Finally, the new tool path is generated by adjusting original tool path. Machining experiments are performed to test the effectiveness of the proposed error compensation method. The error distribution after compensation shows that the average error decreases $74 \%$, and the maximum error (contains overcutting and undercutting) decreases 26\%. Results show that the proposed error compensation method is effective to improve the accuracy for five-axis flank milling.
\end{abstract}

\section{Key words}

Error compensation; Five-axis flank milling; Ruled surface; Tool path modification; Multi-peaks Gaussian fitting. 


\section{Introduction}

Five-axis flank milling plays a significant role in aerospace and transportation industry with the advantages of high accuracy and high efficiency. The increasing demands require different characteristic of the parts. While, it is a trend to achieve higher accuracy for the practical applications, and it is of importance to reduce errors for the manufacturing industry.

There are two main categories of error compensation in recent studies. The first type is based on prediction. These studies focus on the diverse sources of errors during the machining process, analyzing the mechanism of error production, and compensating the sources of errors. Single source of error is considered such as cutting force $[1,2]$, tool deflection [3], dynamic error [4]. Jia and Ma [5] reviewed the mechanism of the formation of the contour error and corresponding methods of compensation. Zhu et al. [6] considered the machine tool as a rigid multi-body system and proposed a model of a geometric error, and the geometric error was compensated by identifying the error sources in the model. Hu et al. [7] proposed an error prediction method considering tool rotation error, machine geometric error, and tool deformation error. The influence of the above three kinds of errors on the geometric error of parts was analyzed by experiments. These models between the errors and sources of errors are analytical or semi-empirical, which need to be calibrated in a laboratory or factory before actual machining. Besides, the sources of errors are tightly coupled to each other, so it is of no accuracy to build a model even if varieties of sources of errors are considered. The other type is based on the actual error distribution. These studies focus on analyzing the characteristic of original errors, and propose some methods to remanufacture at the same part or compensate the errors at the next part. Lo and Hsiao $[8,9]$ proposed error compensation methods firstly, with the beginning of 3-axis end milling for a square block. Cho et al. [10] proposed a method of machining-errors identification, combining the neural networks algorithm and OMM (On Machine Measurement) technology. Poniatowska and Malgorzata [11] obtained the systematic errors by machining a number of surface in the same condition, and compensated systematic errors by 
modifying the tool path. Chen et al. $[12,13]$ proposed a spatial statistical algorithm and an EMD (Empirical Mode Decomposition) algorithm to decompose systematic and random errors based on OMM measuring data, and compensated the systematic errors by modifying NC codes. Jung et al. [14] enhanced the machining accuracy by compensating the volumetric errors of the machine tool. Ma [15] presented an error compensation method for five-axis ball-end milling by reconstructing the CAD model using on-machine-measurement data. Zhu [16] proposed an error compensation method for robotic flanking by globally fitting the cutter envelope.

This paper proposes an error compensation method based on analyzing actual error distribution, and the compensation process is carried out by modifying the original tool path, combining the ideology of single-point offset (SPO) algorithm, which is detailed introduced in Ref [17].

The remainder of this paper is organized as follows. In Section 2, the definition and mathematical expression of ruled surface are presented, and the flow of the proposed method is illustrated. In Section 3, the detailed process of error compensation for five-axis flank milling is proposed. In Section 4, a machining experiment is carried out to validate the proposed method, and the results of the experiment are shown and discussed. Section 5 gives the conclusions of this paper.

\section{Preliminary and flow of proposed method}

\subsection{Mathematical expression of ruled surface}

Ruled surface can be defined as a surface formed by a straight generatrix sweeping along the baseline in 3D space. The mathematical expression of ruled surface by upper and lower alignments is expressed as:

$$
\boldsymbol{s}(u, v)=(1-v) \cdot \boldsymbol{c}_{1}(u)+v \cdot \boldsymbol{c}_{2}(u), u \in[0,1], v \in[0,1]
$$

where $s(u, v)$ is the described ruled surface, $c_{1}(u)$ and $c_{2}(u)$ are the mathematical expression of the upper and lower alignments of the surface, $u$ and $v$ are the location parameter of the surface. 
Set $\boldsymbol{r}_{u}(u, v)$ is the tangent vector in $u$ direction, and $\boldsymbol{r}_{v}(u, v)$ is the tangent vector in $v$ direction. They are the partial derivatives of $s(u, v)$ with respect to $u$ and $v$, and they are calculated as:

$$
\begin{aligned}
& \boldsymbol{r}_{u}(u, v)=\frac{\partial \boldsymbol{s}(u, v)}{\partial u}=-v \cdot \boldsymbol{c}_{1}^{\prime}(u)+v \cdot \boldsymbol{c}_{2}^{\prime}(u) \\
& \boldsymbol{r}_{v}(u, v)=\frac{\partial \boldsymbol{s}(u, v)}{\partial v}=-\boldsymbol{c}_{1}(u)+\boldsymbol{c}_{2}(u)
\end{aligned}
$$

where $\boldsymbol{c}_{1}^{\prime}(u)$ and $\boldsymbol{c}_{2}^{\prime}(u)$ are the first derivatives of $\boldsymbol{c}_{1}(u)$ and $\boldsymbol{c}_{2}(u)$.

The norm vector is the cross product of $\boldsymbol{r}_{u}(u, v)$ and $\boldsymbol{r}_{v}(u, v)$. Assuming that $P\left(x_{0}, y_{0}, z_{0}\right)$ on the surface, its tangent vector in $u$ direction is $\boldsymbol{r}_{u}=\left(x_{u}, y_{u}, z_{u}\right)$, and its tangent vector in $v$ direction is $\boldsymbol{r}_{v}=\left(x_{v}, y_{v}, z_{v}\right)$. The norm vector at $P$ is calculated as:

$$
\boldsymbol{n}=\boldsymbol{r}_{u} \times \boldsymbol{r}_{v}=\left|\begin{array}{ccc}
i & j & k \\
x_{u} & y_{u} & z_{u} \\
x_{v} & y_{v} & z_{v}
\end{array}\right|
$$

Typically, the norm vector represents unit norm vector, and the unit norm vector is calculated as:

$$
\boldsymbol{n}_{u, v}=\frac{\boldsymbol{r}_{u} \times \boldsymbol{r}_{v}}{\left|\boldsymbol{r}_{u} \times \boldsymbol{r}_{v}\right|}
$$




\subsection{Global flows of proposed method}

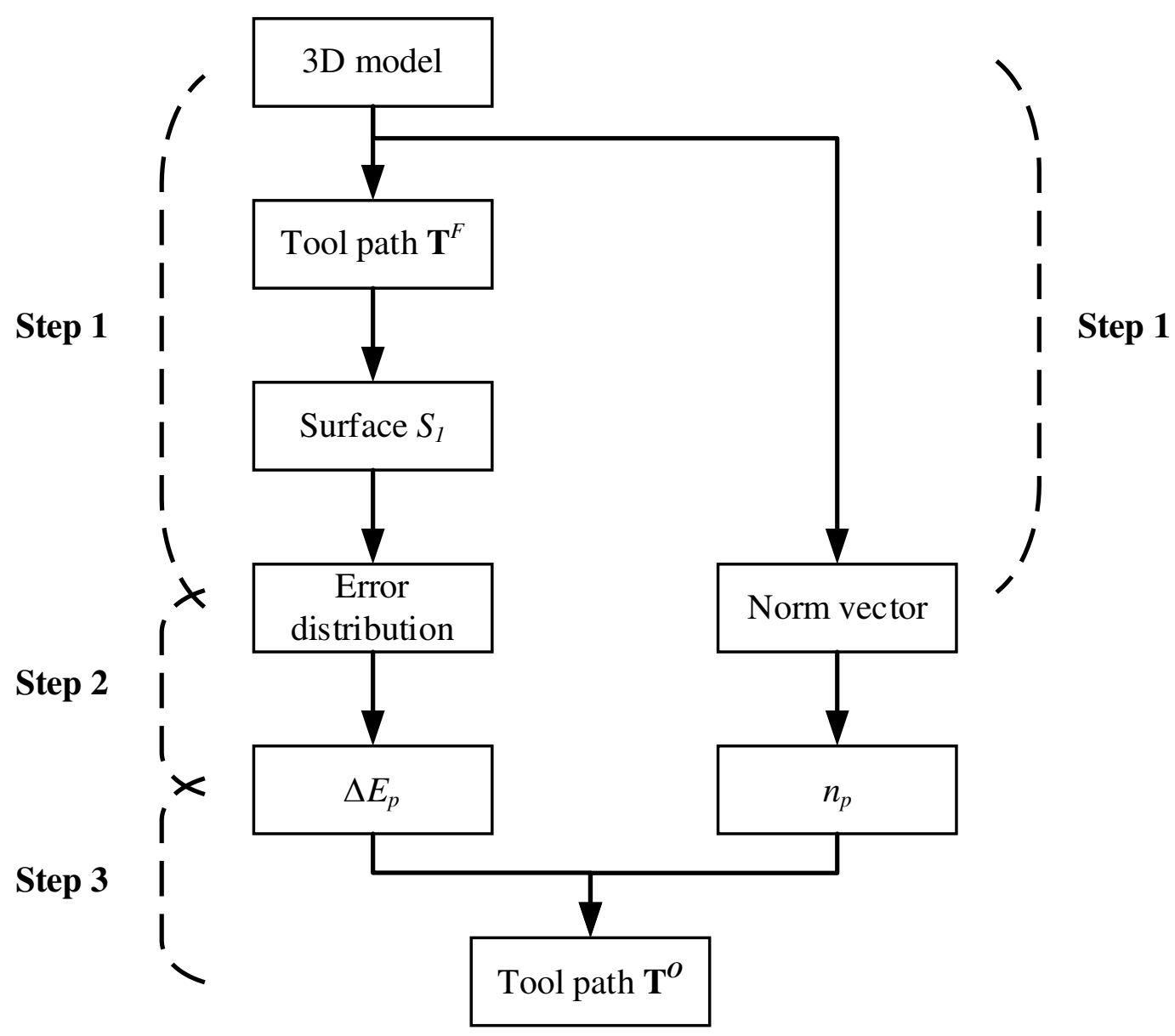

Fig.1 Flowchart of proposed error compensation method.

Fig. 1 shows the flowchart of all the process of the error compensation method. It contains three main steps. Firstly, the error distribution is obtained by calculating the errors between the theoretical points and measuring points. Then, the normal offset value and the norm vector of the tool path are calculated by fitting functions established by the error distribution and the norm vector of the surface. Finally, new tool path is regenerated by offsetting the cutter locations.

\section{The error compensation method}

This paper proposed an improved error compensation method, which can be applied in five-axis flank milling of ruled surface. 


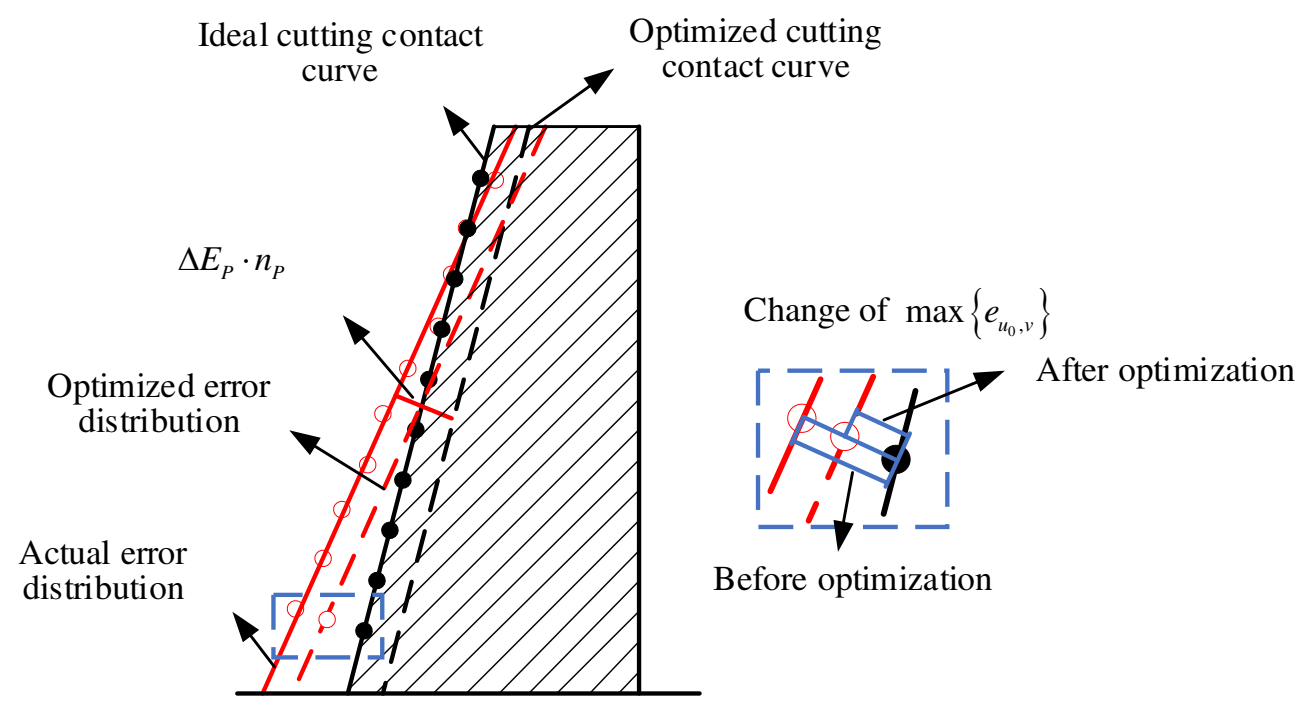

Fig. 2 Comparison of error distribution at $u_{0}$ section before and after compensation.

The tool path is generated by varieties of methods based on the cutting contact curve. In ideal conditions, the cutting contact curve of the cutter is closed to the straight generatrix of the surface infinitely. However, the actual machining process is complicated, and the formation of geometric error is controlled by varieties of conditions. In this paper, the model between error sources and actual error distribution is regarded as a black box, it is assumed that the error distribution is constant under the same conditions. On the one hand, the tool deformation accounts for a small proportion among all error sources [7]. On the other hand, the errors caused by cutting force is a small proportion of the machine centers [18] and the tool deformation can be neglected because of the slight material removal and low cutting force in the finish machining process. Therefore, the error distribution is nearly linear in the $v$ direction. Further, the variation of cutter location hardly influences the black box. However, the cutter orientation affects the kinematics characteristics of the machine tool significantly, which will change the form of error distribution. So, the cutter orientation stays constant in this paper. As shown in Fig.2, the $\max \left\{e_{u_{0}, v}\right\}$ can be reduced at $u_{0}$ by offsetting the contact curve $\Delta E_{p} \cdot n_{p}$, which means offsetting the original tool path $\Delta E_{p} \cdot n_{p}$ can 
reduce $\max \left\{e_{u_{0}, v}\right\}$.

\subsection{Calculation of normal offset value and normal vector of the tool path}

It is fundamental to acquire actual error distribution of the machined surface before error compensation. Error distribution is obtained by calculating the deviation between the theoretical points and the measured points.

The point-to-surface distance is replaced by error in the paper. Error is the basis of the accuracy of the machined surface and the quality of the tool path generation. The coordinates of theoretical points at are expressed as $P_{u, v}^{T}=\left(x_{u, v}^{T}, y_{u, v}^{T}, z_{u, v}^{T}\right)$, while the corresponding coordinates of measured points are expressed as $P_{u, v}^{M}=\left(x_{u, v}^{M}, y_{u, v}^{M}, z_{u, v}^{M}\right)$. As shown in Fig. $3, e_{u_{0}, v_{0}}$ at $\left(u_{0}, v_{0}\right)$ is calculated as

$$
e_{u_{0}, v_{0}}=\sqrt{\left(x_{u_{0}, v_{0}}^{M}-x_{u_{0}, v_{0}}^{T}\right)^{2}+\left(y_{u_{0}, v_{0}}^{M}-y_{u_{0}, v_{0}}^{T}\right)^{2}+\left(z_{u_{0}, v_{0}}^{M}-z_{u_{0}, v_{0}}^{T}\right)^{2}}
$$

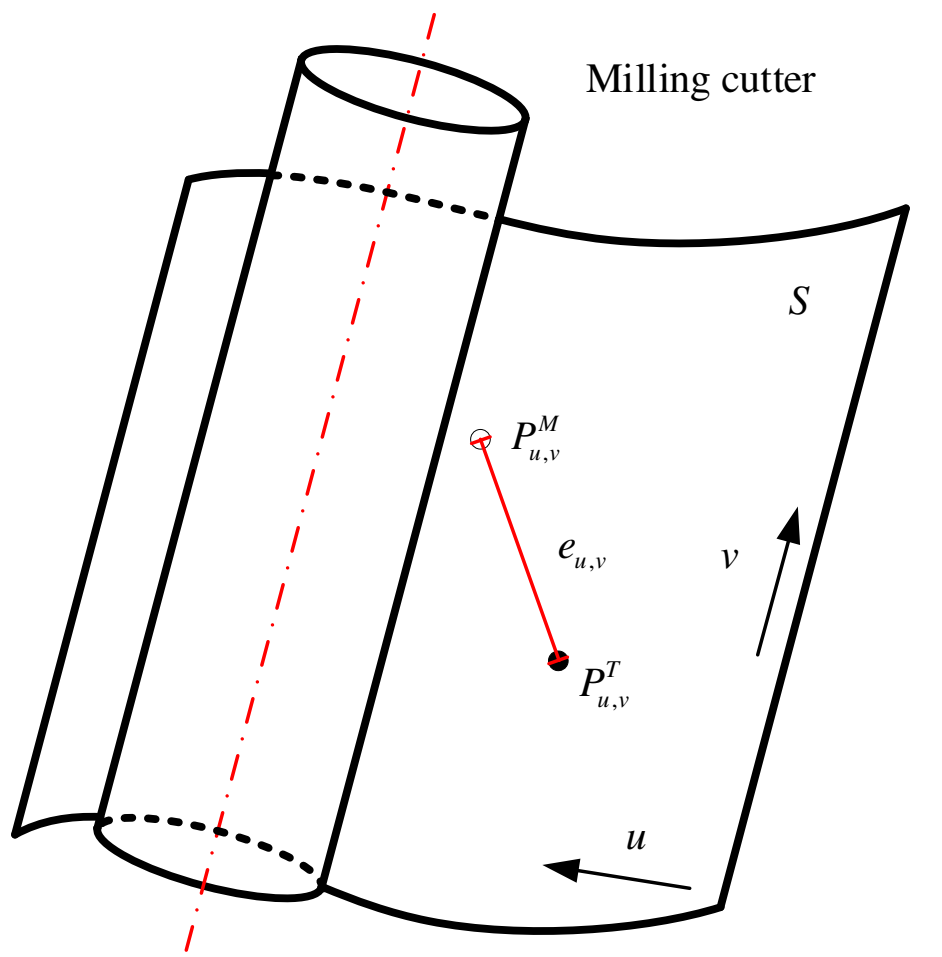

Fig. 3. Distance from measuring point to theoretical point. 
According to the feature of error distribution at surface $S$, an actual cutting contact curve can be generated by fitting all $P_{u_{0}, v}^{M}$ points at $u_{0}$, using least squares fitting methods. Objective fitting function is as following :

$$
e_{u_{0}, v}=\hat{k}_{u_{0}} \cdot v+\hat{b}_{u_{0}}
$$

where $\hat{k}_{u_{0}}$ and $\hat{b}_{u_{0}}$ are coefficients of the objective fitting function in $u_{0} . \mathbf{e}_{u, 0.5}$ represents all the errors when $v=0.5$.

Normal vectors of the surface can be calculated by substitute $v=0.5$ into formula (4).

Cutter location and cutter orientation are generated by variety conditions in CAM (Computer-Aided Manufacture) software. The actual cutting contact curve cannot be depicted by error distribution directly, therefore, it is important to calculate offset value $\Delta E_{p}$. Actually, the cutter locations of the tool path are not on the surface or they cannot be transferred by translation easily. What' more, the norm vector of the cutter locations $n_{p}$ is difficult to be calculated in geometry. In this paper, multi-peaks Gaussian fitting method is applied to calculate $\Delta E_{p}$ and $n_{p}$ with a continuously increasing coordinate value. In addition, both the tool path and $\mathbf{e}_{u, 0.5}$ must be projected in a plane and the cartesian coordinates of $\mathbf{e}_{u, 0.5}$ must be offset a radius of cutter before fitting.

$$
\left(x_{u_{0}}^{\prime}, y_{u_{0}}^{\prime}, z_{u_{0}}^{\prime}\right)=\left(x_{u_{0}}-R \cdot i_{u_{0}}, y_{u_{0}}-R \cdot j_{u_{0}}, z_{u_{0}}-R \cdot k_{u_{0}}\right)
$$

where $\left(x_{u_{0}}^{\prime}, y_{u_{0}}^{\prime}, z_{u_{0}}^{\prime}\right)$ is the cartesian coordinate at $\left(u_{0}, v_{0}\right),\left(i_{u_{0}}, j_{u_{0}}, k_{u_{0}}\right)$ is the normal vector at $\left(u_{0}, v_{0}\right)$, and $R$ is the radius of the cutter.

The objective equation of multi-peaks Gaussian fitting is expressed as:

$$
Y=\sum_{q=1}^{K} a_{q} \cdot e^{\left(\frac{X-b_{q}}{c_{q}}\right)^{2}}
$$

where $Y$ and $X$ are variables of the fitting equation, $K$ is the number of peaks of the data, $a_{q}, b_{q}$, and $c_{q}$ are coefficients of the fitting equation. 


\subsection{Tool path generation}

Generally, the tool path containing cutter locations and cutter orientations is generated by CAM software, and then the post-processor converts the tool path into a NC program which can be recognized by a certain machine tool. The tool path regeneration is realized by modifying cutter locations.

Assuming the former tool path $\mathbf{T}^{F}=\left[\begin{array}{c:c}x_{1}^{F}, y_{1}^{F}, z_{1}^{F} & \alpha_{1}^{F}, \beta_{1}^{F}, \chi_{1}^{F} \\ x_{2}^{F}, y_{2}^{F}, z_{2}^{F} & \alpha_{2}^{F}, \beta_{2}^{F}, \chi_{2}^{F} \\ \mathbf{M} & \mathrm{M} \\ x_{L}^{F}, y_{L}^{F}, z_{L}^{F} & \alpha_{L}^{F}, \beta_{L}^{F}, \chi_{L}^{F}\end{array}\right]$, the optimized tool path can be calculated as:

$$
\mathbf{T}_{O}=\mathbf{T}_{F}-\Delta E_{p} \cdot n_{p}=\left[\begin{array}{c:c}
x_{1}^{F}-\Delta E_{1} \cdot i_{1}, y_{1}^{F}-\Delta E_{1} \cdot j_{1}, z_{1}^{F}-\Delta E_{1} \cdot k_{1} & \alpha_{1}^{F}, \beta_{1}^{F}, \chi_{1}^{F} \\
x_{2}^{F}-\Delta E_{2} \cdot i_{2}, y_{2}^{F}-\Delta E_{2} \cdot j_{2}, z_{2}^{F}-\Delta E_{2} \cdot k_{2} & \alpha_{2}^{F}, \beta_{2}^{F}, \chi_{2}^{F} \\
\mathrm{M} & \mathrm{M} \\
x_{L}^{F}-\Delta E_{L} \cdot i_{L}, y_{L}^{F}-\Delta E_{L} \cdot j_{L}, z_{L}^{F}-\Delta E_{L} \cdot k_{L} & \alpha_{L}^{F}, \beta_{L}^{F}, \chi_{L}^{F}
\end{array}\right]
$$

where $\left(x_{L}^{F}, y_{L}^{F}, z_{L}^{F}\right)$ is the coordinate of the $L$ th cutter location, $\left(\alpha_{L}^{F}, \beta_{L}^{F}, \chi_{L}^{F}\right)$ is the $L$ th cutter orientation, $\Delta E_{L}$ is the offset value after fitting, and $n_{L}=\left(i_{L}, j_{L}, k_{L}\right)$, is the offset vector after fitting.

\section{Experiment and results}

\subsection{Setup of experiment}

To verify the validation and accuracy of the proposed error compensation method of ruled surface, a machining experiment is conducted on a five-axis machine center DMU-60 mono BLOCK with rotary axes B and C. The ruled surface is designed and the tool path is generated by the CAM software Unigraphics (UG). The NC codes are generated by Unigraphics (UG) with post-processor provided by manufacturer of the machine center. 


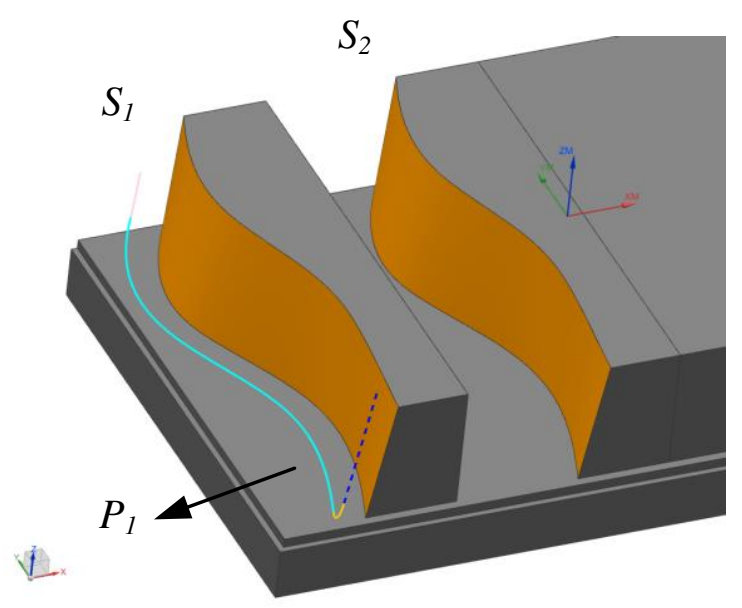

Fig. 4 Designed surfaces and tool path.

As shown in Fig.4, $S_{1}$ and $S_{2}$ are the completely same ruled surfaces in geometry, and $S_{2}$ is an offset surface of $S_{1}$ in $X$ direction. $P_{1}$ is the tool path of $S_{1}$ and generated by Unigraphics (UG). Upper and lower alignments of $S_{l}$ are cubic spline curves, and their control points are at list in Tab. 1.

Tab.1 Coordinate of control points of alignments.

\begin{tabular}{cc}
\hline Upper alignment/mm & Lower alignment/mm \\
\hline$(28,3,0)$ & $(35,2.9,40)$ \\
$(38,30,0)$ & $(38,30,40)$ \\
$(40,62,0)$ & $(43,62,40)$ \\
$(20,94,0)$ & $(28,94,40)$ \\
$(8,126,0)$ & $(18,126,40)$ \\
$(28,158,0)$ & $(31,158,40)$ \\
\hline
\end{tabular}

In order to reduce the non-synchronization of cutter location and cutter orientation, feedrate is limited relatively [19]. Finish machining conditions are listed in Tab. 2. 
Tab.2 Finish machining conditions of the experiment.

\begin{tabular}{cc}
\hline Machining conditions & value \\
\hline Diameter of cutter & $20 \mathrm{~mm}$ \\
Material of blank & Aluminum alloy 5250 \\
Feedrate & $120 \mathrm{~mm} / \mathrm{min}$ \\
Spindle speed & $1500 \mathrm{rpm}$ \\
Direction of material removal & Radial of the cutter \\
\hline
\end{tabular}

The machining process is shown in Fig. 5.

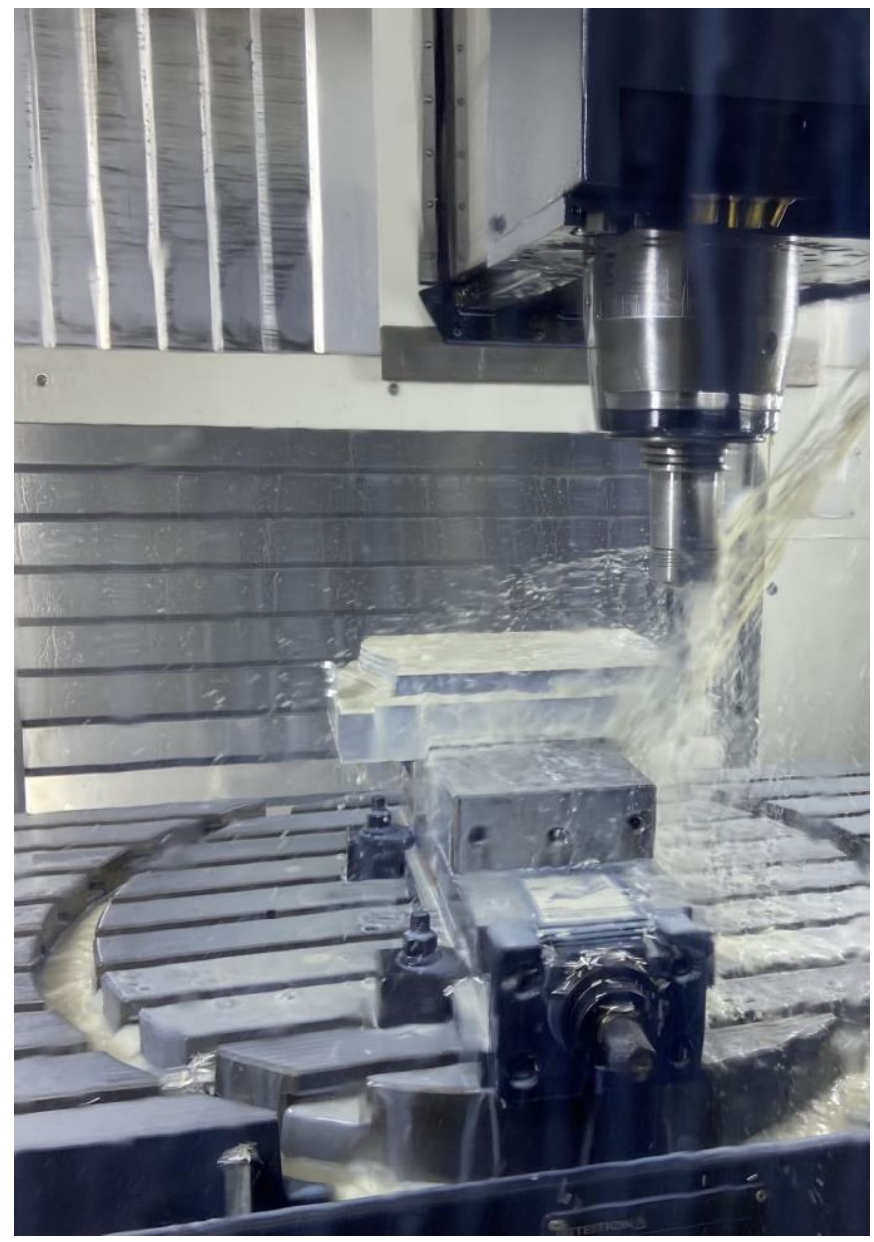

Fig. 5 The machining process of the part.

The offline measurement experiment is carried out on a Daisy CMM (coordinate measuring machine, $\left.\mathrm{MPE}_{\mathrm{E}}=2.0+\mathrm{L} / 300 \mu \mathrm{m}\right)$, which was equipped with a touch probe with a diameter of $4 \mathrm{~mm}$. 
Surface $S_{1}$ was machined firstly, and then it is transferred to the CMM after finishing process. $100 \times 10$ iso-parametric measurement points are distributed in surface $S_{1}$. The machining error distribution was shown in Fig. 6 (a).

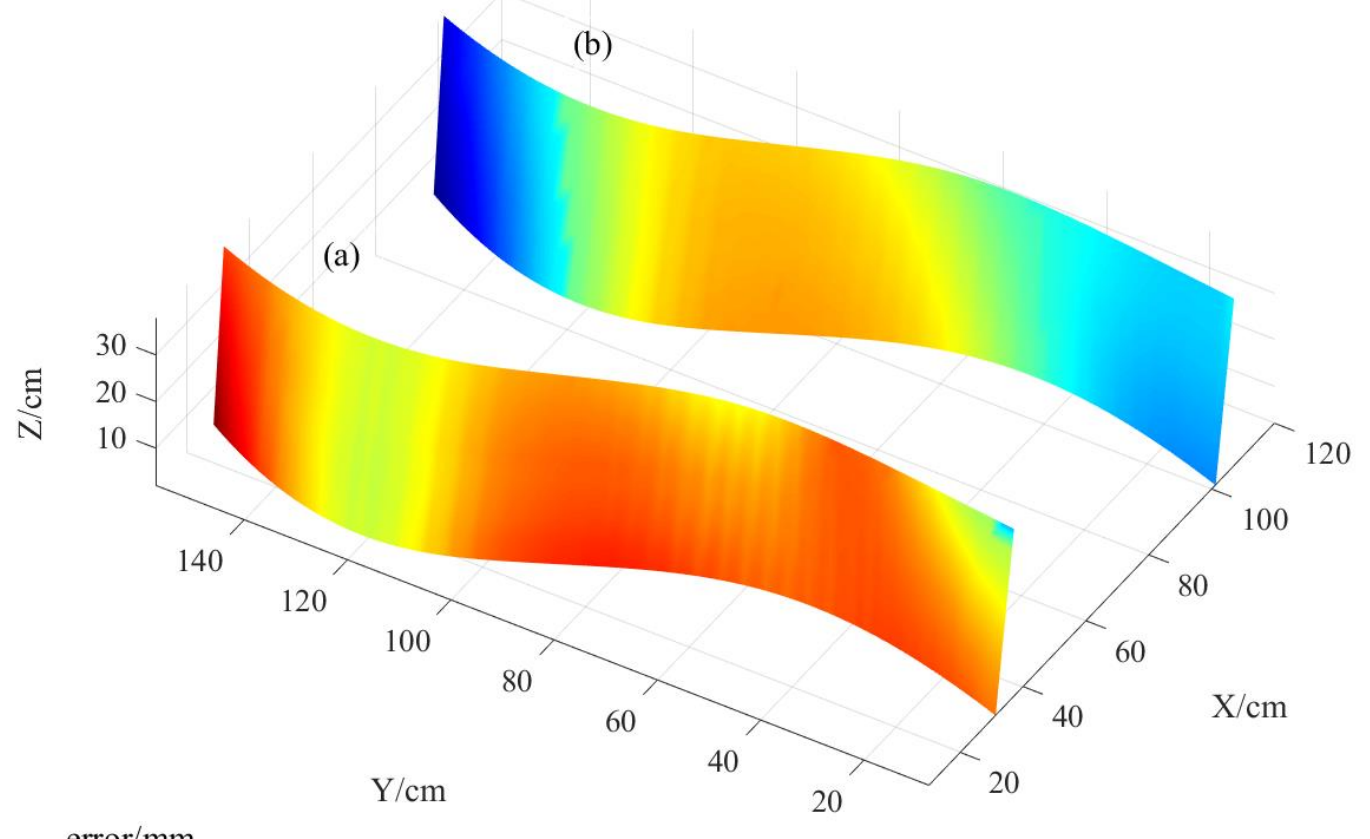

error/mm

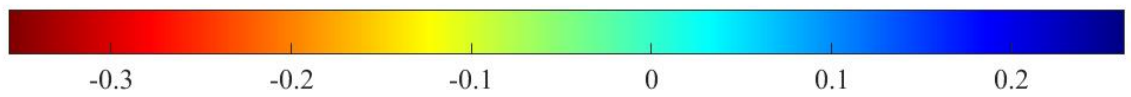

Fig. 6 The error distribution of the surfaces: (a) The errors distribution of $S_{1}$. (b) The errors distribution of $S_{2}$.

In Fig. 6, the negative value of the color bar denotes overcut, and the positive value denotes undercut.

According to section 2.1, 100 fitting lines are calculated and the average coefficient of determination is 0.9015 , which indicated that the linear fitting meet the assumption in Fig. 2. 100 groups of $e_{u_{0}, 0.5}$ are calculated according to formula (6), and the norm vector $n_{u_{0}, 0.5}=\left(i_{u_{0}, 0.5}, j_{u_{0}, 0.5}, k_{u_{0}, 0.5}\right)$ are calculated according to formula (4). 4peakes Gaussian fitting method is utilized to fit $\mathbf{e}_{u, 0.5}, \mathbf{i}_{u, 0.5}, \mathbf{j}_{u, 0.5}$, and $\mathbf{k}_{u, 0.5}$, with the increasing of $Y$ coordinate in this experiment, and Fig. 7 shows the fitting results. The coefficients of the 4 fitting functions are listed in Appendix A. And $\mathbf{T}^{O}$ is generated 
according to formula (8) and extracted $\mathbf{T}^{F}$. At last, $\mathbf{T}^{O}$ is translated $70 \mathrm{~mm}$ in $X$ direction, to prevent interference of the two surfaces.

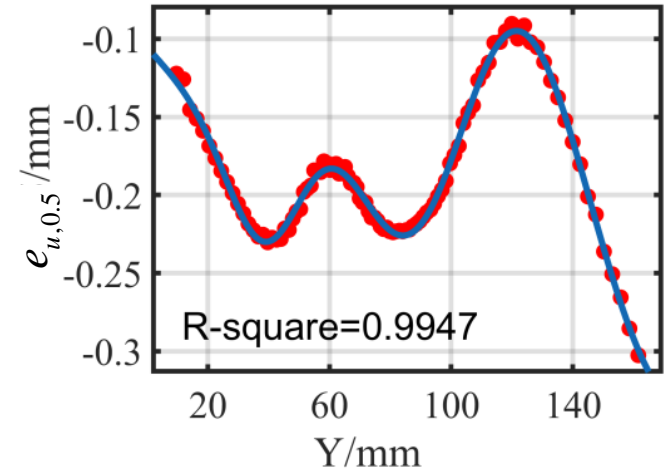

(a)

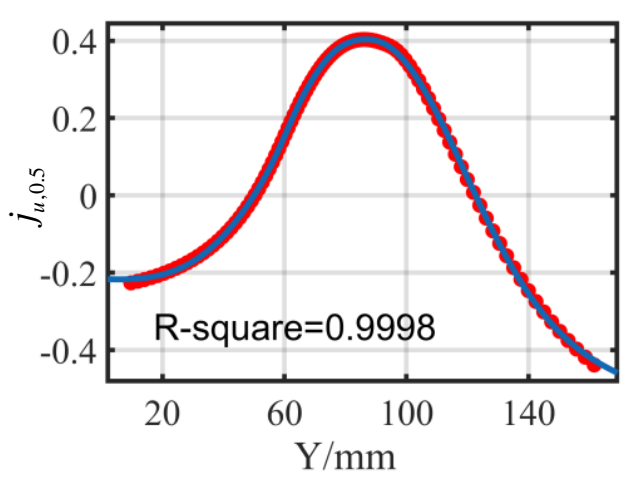

(c)

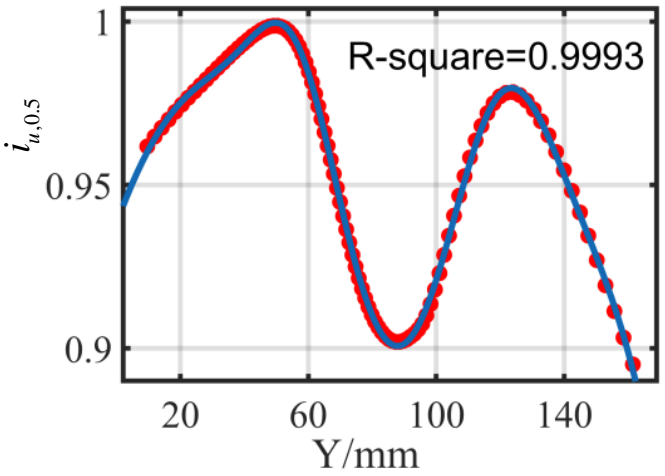

(b)

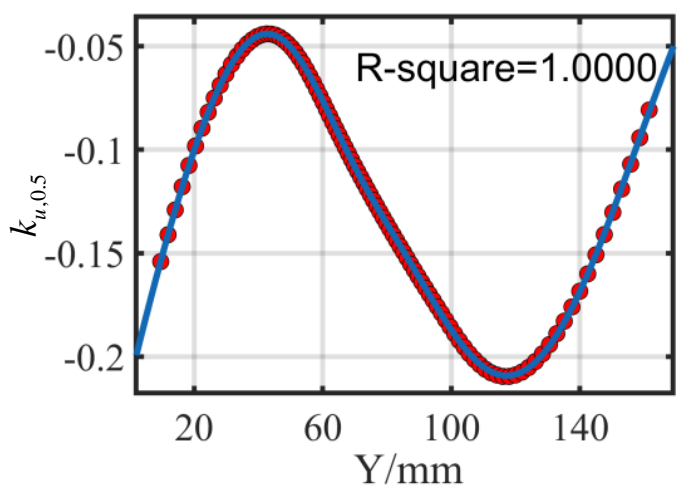

(d)

Fig. 7 Results of 4-peaks Gaussian fitting: (a) The fitting result of $\mathbf{e}_{u, 0.5}$. (b) The fitting result of $\mathbf{i}_{u, 0.5}$. (c) The fitting result of $\mathbf{j}_{u, 0.5}$. (d) The fitting result of $\mathbf{k}_{u, 0.5}$.

The final NC codes are got by converting the $\mathbf{T}^{O}$ by the post-processor. Other machining conditions of $S_{2}$ are the same as the machining conditions of $S_{1}$.

\subsection{Result of experiment}

Surface $S_{2}$ is also measured on the Daisy CMM. 


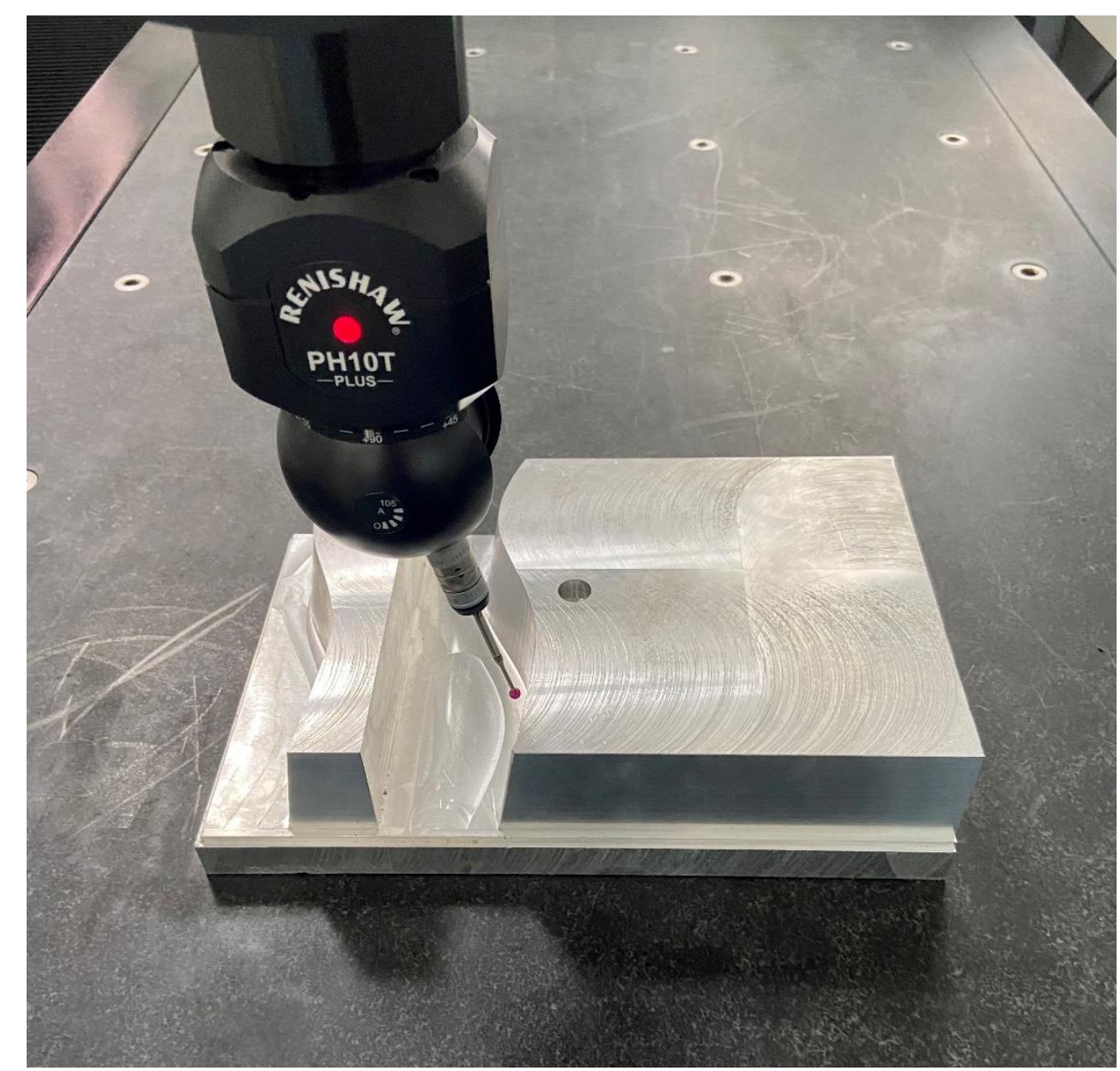

Fig. 8 Measurement of the two surfaces on the CMM.

The error distribution before and after optimization by the proposed error compensation method are presented in Fig. 8. The histograms of the 1000 errors of the surface $S_{1}$ and the surface $S_{2}$ are shown in Fig. 9 (a) and (b). By comparing the two figures, it can be seen that the average error is $-0.1881 \mathrm{~mm}$ before optimization, and the average error is reduced to $-0.0486 \mathrm{~mm}$ after optimization. The average error is reduced by $74 \%$ with error compensation. Besides, the maximum error (including overcut and undercut) is decreased from $-0.3569 \mathrm{~mm}$ to $0.2626 \mathrm{~mm}$, with an improvement of $26 \%$. The range of the errors can be expressed as:

$$
\text { Range }=\max \{\mathbf{e}\}-\min \{\mathbf{e}\}
$$

where e denotes all the errors in the surface. The range of the errors is $0.4376 \mathrm{~mm}$ before compensation, and it changes to $0.4481 \mathrm{~mm}$ after compensation, which indicates that the range of the errors maintains during the compensation is constant, what's more, 
illustrates the correctness of the proposed method in Fig. 2.

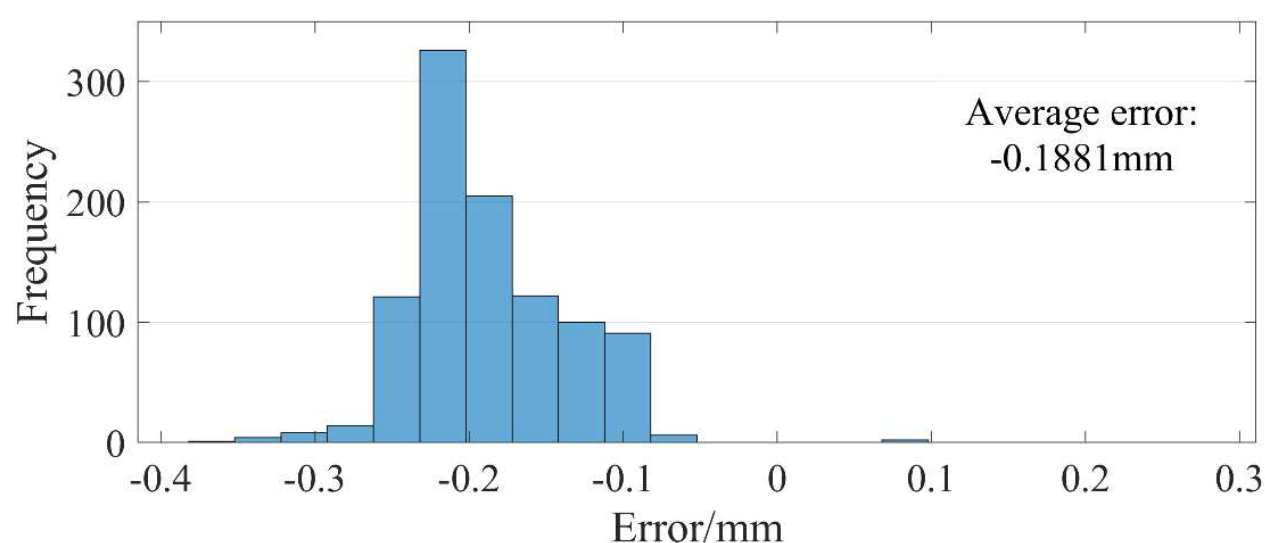

(a)

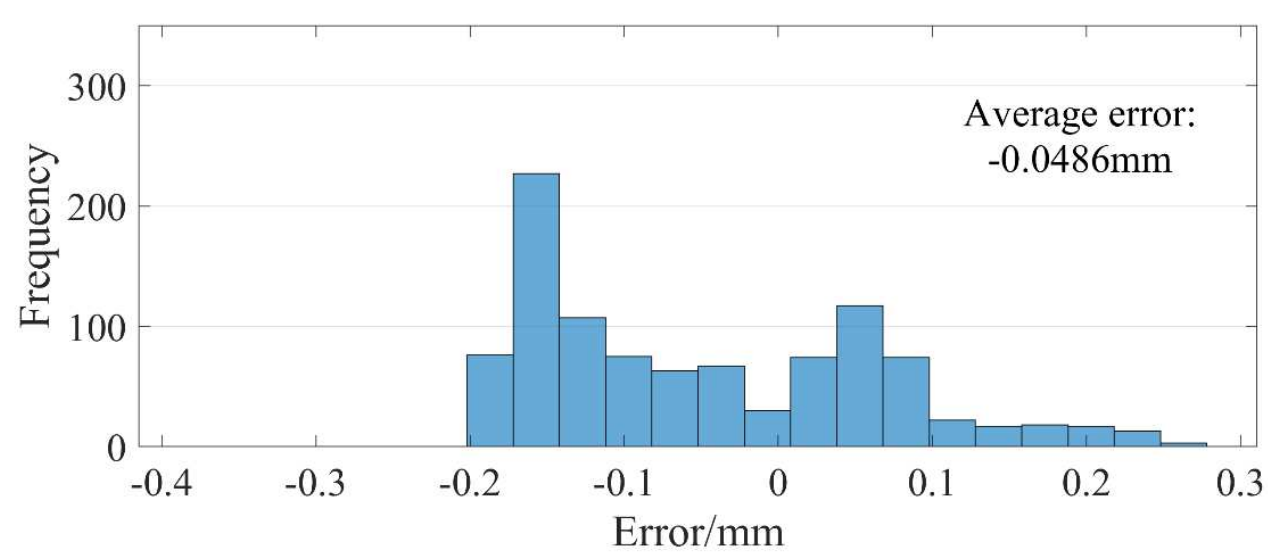

(b)

Fig. 9 Histogram of the errors: (a) The errors of $S_{1}$. (b) The errors of $S_{2}$.

\section{Conclusions}

In this paper, a method of error compensation for the ruled surface is presented to increase the machining quality by modified the tool path based on the error data of the machined part. At first, the errors at the middle of the straight generatrix on the machined surface are calculated by linear fitting of the errors distributed in $u_{0}$, and the corresponding normal vectors are calculated by given formula based on the mathematical expression of the surface. Then, the errors and the corresponding normal vectors calculated in the last step are fitted with the increasing of a continuously 
increasing coordinate value. The value of normal offset of the tool path is calculated by the fitting function of the errors, and the normal vector of offset of the tool path is calculated by the fitting function of the corresponding normal vectors. Finally, the tool path with compensation is generated by the original tool path, the value and normal vector of offset of the tool path. The method is validated by an experiment carried out on a five-axis machine center. Results show that the average errors decrease $74 \%$, and the maximum error decreases $26 \%$.

Despite the improvements in the work, there are also some shortcomings during the process. Although the measurement data of CMM is much precise than that of OMM, the errors of inspecting could not be eliminated completely. Moreover, the random error is over-compensated in this method unless measuring massive parts machined in the same conditions.

In future work, the tool orientation will be considered and improved in the tool path regeneration by analyzing the data deeply.

\section{a. Funding}

This study is supported by the National Natural Science Foundation of China (No.51675378).

\section{b. Conflicts of interest/Competing interests}

The authors declare that they have no known competing financial interests or personal relationships that could have appeared to affect the work reported in the paper.

\section{c. Availability of data and material}

The errors and cutter locations before and after error compensation are available in Supplementary Material. 


\section{d. Code availability}

Not applicable.

\section{e. Ethics approval}

Not applicable.

\section{f. Consent to participate}

All the authors, namely Gaiyun He, Chenglin Yao, Yicun Sang, and Yichen Yan have contented to participate in the paper and agreed to submit the manuscript.

\section{g. Consent for publication}

All the authors, namely Gaiyun He, Chenglin Yao, Yicun Sang, and Yichen Yan have contented to publish the manuscript after peer review.

\section{h. Authors' contributions}

Gaiyun He: Supervision, Resources, Funding acquisition, Conceptualization;

Chenglin Yao: Conceptualization, Methodology, Formal analysis, Software, Validation, Original Draft Writing;

Yicun Sang: Software, Conceptualization, Writing-Reviewing;

Yichen Yan: Investigation, Data Curation, Writing-Editing.

\section{References}

[1] Omar O, El-Wardany T, Ng E, Elbestawi, M.(2007). An improved cutting force and surface topography prediction model in end milling. Int J Mach Tools Manuf. http://doi.org/10.1016/j.ijmachtools.2006.08.021

[2] Sui S, Li Y, Shao W, Feng P.(2015). Tool path generation and optimization 
method for pocket flank milling of aircraft structural parts based on the constraints of cutting force and dynamic characteristics of machine tools. Int J Adv Manuf Technol. http://doi.org/10.1007/s00170-015-8050-x

[3] Habibi M, Arezoo B, Vahebi N. (2011). Tool deflection and geometrical error compensation by tool path modification. Int J Mach Tools Manuf. http://doi.org/10.1016/j.ijmachtools.2011.01.009

[4] Dumanli A, Sencer B.(2019). Pre-compensation of servo tracking errors through data-based reference trajectory modification. CIRP Ann-Manuf Technol. http://doi.org/10.1016/j.cirp.2019.03.017

[5] Jia Z, Ma J, Song D, Wang F, Liu W. (2018). A review of contouring-error reduction method in multi-axis CNC machining. Int J Mach Tools Manuf. http://doi.org/10.1016/j.ijmachtools.2017.10.008

[6] Zhu S, Ding G, Qin S, Lei J, Zhuang L, Yan K. (2012). Integrated geometric error modeling, identification and compensation of CNC machine tools. Int J Mach Tools Manuf. http://doi.org/10.1016/j.ijmachtools.2011.08.011

[7] Hu W, Cao Y, Yang J, Shang H, Wang W. (2016). An error prediction model of NC machining process considering multiple error sources. Int J Adv Manuf Technol. http://doi.org/10.1007/s00170-016-9867-7

[8] Lo C-C, Hsiao C-Y. (1998). CNC machine tool interpolator with path compensation for repeated contour machining. Comput-Aided Des. http://doi.org/10.1016/S0010-4485(97)00053-5

[9] Lo C-C, Hsiao C-Y. (1997). A method of tool path compensation for repeated machining process. Int J Mach Tools Manuf. http://doi.org/10.1016/S08906955(97)00049-7

[10]Cho M-W, Kim G-H, Seo T-I, Hong Y-C, Cheng H-H. (2006). Integrated machining error compensation method using OMM data and modified PNN algorithm. Int J Mach Tools Manuf. http://doi.org/10.1016/j.ijmachtools.2005.10.002 [11] Poniatowska, Malgorzata. (2015). Free-form surface machining error compensation applying 3D CAD machining pattern model. Comput-Aided Des. http://doi.org/10.1016/j.cad.2014.12.003 
[12]Chen Y, Gao J, Deng H, Zheng D, Chen X, Kelly R. (2013). Spatial statistical analysis and compensation of machining errors for complex surfaces. Precis Eng-J Int Soc Precis Eng Nanotechnol. http://doi.org/10.1016/j.precisioneng.2012.08.003 [13]Chen Y, Tang H, Tang Q, Zhang A, Chen D, Li K. (2018). Machining error decomposition and compensation of complicated surfaces by EMD method. Measurement. http://doi.org/10.1016/j.measurement.2017.11.027 [14]Jung J-H, Choi J-P, Lee S-J. (2006). Machining accuracy enhancement by compensating for volumetric errors of a machine tool and on-machine measurement. $\mathbf{J}$ Mater Process Technol. http://doi.org/10.1016/j.jmatprotec.2004.12.014 [15] Ma W, He G, Han J, Xie Q. (2020). Error compensation for machining of sculptured surface based on on-machine measurement and model reconstruction. Int $\mathbf{J}$ Adv Manuf Technol. http://doi.org/10.1007/s00170-019-04862-0

[16]Xiong G, Li Z, Ding Y, Zhu L. (2020). A closed-loop error compensation method for robotic flank milling. Robot Comput-Integr Manuf. http://doi.org/10.1016/j.rcim.2019.101928 [17]Liu X. (1995). Five-axis NC cylindrical milling of sculptured surfaces. ComputAided Des. http://doi.org/10.1016/0010-4485(95)00005-4

[18] Shen H, Fu J, He Y, Yao X. (2012). On-line asynchronous compensation methods for static/quasi-static error implemented on CNC machine tools. Int J Mach Tools Manuf. http://doi.org/10.1016/j.ijmachtools.2012.04.003

[19] Wan X, Xiong C, Hua L. (2011). Synchronous adjustment of milling tool path based on the relative deviation. J Manuf Sci Eng-Trans ASME. http://doi.org/10.1115/1.4004201

\section{Appendix A. The coefficients of 4-peaks Gaussian fitting}

The coefficients of 4-peaks Gaussian fitting of $\mathbf{e}_{u, 0.5}$ are listed in Tab. A1.

Tab. A1 The coefficients of $\mathbf{e}_{u, 0.5}$. 


\begin{tabular}{lll}
\hline $\mathrm{a}_{1}=-0.4496$ & $\mathrm{~b}_{1}=39.11$ & $\mathrm{c}_{1}=35.69$ \\
\hline $\mathrm{a}_{2}=-0.1191$ & $\mathrm{~b}_{2}=75.31$ & $\mathrm{c}_{2}=11.86$ \\
$\mathrm{a}_{3}=-0.3398$ & $\mathrm{~b}_{3}=93.25$ & $\mathrm{c}_{3}=19.91$ \\
$\mathrm{a}_{4}=-0.7153$ & $\mathrm{~b}_{4}=180.6$ & $\mathrm{c}_{4}=46.19$ \\
\hline
\end{tabular}

The coefficients of 4-peaks Gaussian fitting of $\mathbf{i}_{u, 0.5}$ are listed in Tab. A2.

Tab. A2 The coefficients of $\mathbf{i}_{u, 0.5}$.

\begin{tabular}{ccc}
\hline $\mathrm{a}_{1}=-3765$ & $\mathrm{~b}_{1}=58.04$ & $\mathrm{c}_{1}=32.07$ \\
\hline $\mathrm{a}_{2}=0.9248$ & $\mathrm{~b}_{2}=120.4$ & $\mathrm{c}_{2}=53.55$ \\
$\mathrm{a}_{3}=0.5127$ & $\mathrm{~b}_{3}=181.3$ & $\mathrm{c}_{3}=36.47$ \\
$\mathrm{a}_{4}=0.9134$ & $\mathrm{~b}_{4}=5.818$ & $\mathrm{c}_{4}=55.20$ \\
\hline
\end{tabular}

The coefficients of 4-peaks Gaussian fitting of $\mathbf{j}_{u, 0.5}$ are listed in Tab. A3. Tab. A3 The coefficients of $\mathbf{j}_{u, 0.5}$.

\begin{tabular}{ccc}
\hline $\mathrm{a}_{1}=-2.229$ & $\mathrm{~b}_{1}=20.29$ & $\mathrm{c}_{1}=14.94$ \\
\hline $\mathrm{a}_{2}=0.6258$ & $\mathrm{~b}_{2}=0.3222$ & $\mathrm{c}_{2}=1.216$ \\
$\mathrm{a}_{3}=-2.473$ & $\mathrm{~b}_{3}=0.1561$ & $\mathrm{c}_{3}=0.487$ \\
$\mathrm{a}_{4}=2.624$ & $\mathrm{~b}_{4}=0.1535$ & $\mathrm{c}_{4}=0.503$ \\
\hline
\end{tabular}

The coefficients of 4-peaks Gaussian fitting of $\mathbf{k}_{u, 0.5}$ are listed in Tab. A4.

Tab. A4 The coefficients of $\mathbf{k}_{u, 0.5}$.

\begin{tabular}{lll}
\hline $\mathrm{a}_{1}=-0.3018$ & $\mathrm{~b}_{1}=-27.20$ & $\mathrm{c}_{1}=44.82$ \\
\hline $\mathrm{a}_{2}=-0.0506$ & $\mathrm{~b}_{2}=70.27$ & $\mathrm{c}_{2}=22.57$ \\
$\mathrm{a}_{3}=-0.1961$ & $\mathrm{~b}_{3}=112.5$ & $\mathrm{c}_{3}=37.60$ \\
$\mathrm{a}_{4}=-0.0587$ & $\mathrm{~b}_{4}=148.1$ & $\mathrm{c}_{4}=26.96$ \\
\hline
\end{tabular}




\section{Supplementary Files}

This is a list of supplementary files associated with this preprint. Click to download.

- AMTSupplementaryMaterial.xlsx 